\title{
A 63 signature genes prediction system is effective for glioblastoma prognosis
}

\author{
YANG ZHANG, JIAMING XU and XIANGDONG ZHU \\ Department of Neurosurgery, The Second Affiliated Hospital of Zhejiang University \\ School of Medicine, Hangzhou, Zhejiang 310009, P.R. China
}

Received May 18, 2017; Accepted January 10, 2018

DOI: $10.3892 /$ ijmm.2018.3422

\begin{abstract}
The present study aimed to explore possible prognostic marker genes in glioblastoma (GBM). Differentially expressed genes (DEGs) were screened by comparing microarray data of tumor and normal tissue samples from The Cancer Genome Atlas (TCGA) and the Gene Expression Omnibus (GEO) dataset GSE22866. Subsequently, the prognosis-associated DEGs were screened via Cox regression analysis, followed by construction of gene/protein/pathway interaction networks of these DEGs by calculating the correlation coefficient between the DEGs. Next, a prognostic prediction system was constructed using Bayes discriminant analysis, which was validated by the microarray data of samples from patients with good and bad prognosis from the TCGA and Chinese Glioma Genome Atlas (CGGA), as well as the GEO dataset. Finally, a co-expression network of the signature genes in the prediction system was constructed in combination with the significant pathways. A total of 288 overlapping DEGs (false discovery rate $<0.5$ and $\log 2$ of fold changel $>1$ ) were screened, 123 of which were identified to be associated with the prognosis of GBM patients. The co-expression network of these prognosis-associated DEGs included 1405 interactions and 112 DEGs, and 6 functional modules were identified in the network. The prognostic prediction system was comprised of 63 signature genes with a specificity value of 0.929 and a sensitivity value of 0.948 . GBM samples with good and bad prognosis in the TCGA, CGGA and GEO datasets were distinguishable by these signature genes $\left(\mathrm{P}=1.33 \times 10^{-6}, 1.63 \times 10^{-4}\right.$ and 0.00534 , respectively). The co-expression network of signature genes with significant pathways was comprised of 56 genes and 361 interactions. Protein kinase $\mathrm{C} \gamma$ (PRKCG), protein kinase $\mathrm{C} \beta$ (PRKCB) and calcium/calmodulin-dependent
\end{abstract}

Correspondence to: Dr Xiangdong Zhu, Department of Neurosurgery, The Second Affiliated Hospital of Zhejiang University School of Medicine, 88 Jiefang Road, Hangzhou, Zhejiang 310009, P.R. China

E-mail:xdzhu2015@sina.com

Key words: glioblastoma, differentially expressed genes, co-expression network, prognosis, PRKCG, PRKCB protein kinase II $\alpha$ (CAMK2A) were important genes in the network, and based on the expression of these genes, it was possible to distinguish between samples with significantly different survival risks. In the present study, an effective prognostic prediction system for GBM patients was constructed and validated. PRKCG, PRKCB and CAMK2A may be potential prognostic factors for GBM.

\section{Introduction}

Glioblastoma (GBM) ranks as the most common malignant brain tumor type in adults. Despite the low incidence of $\sim 6$ out of 100,000 individuals, the mortality rate of GBM is relatively high (1). Patients with GBM suffer not only from headaches, seizures and focal deficits, but also exhibit personality and behavioral changes (2). GBM has a poor prognosis with relatively low survival estimates and the 5-year survival rate is $<5 \%$ (3).

Molecular-based therapies are considered to be breakthrough measures for GBM (4). Various prognostic markers have been identified in GBM, including overexpressed epidermal growth factor receptor (EGFR) and mutated tumor protein p53 (5). The EGFR vIII mutation is a GBM-specific therapeutic target which is present in $50 \%$ of EGFR gene-amplified GBM (6). Phosphatase and tensin homolog was reported to be mutated in 5-40\% tumor tissues of GBM patients and is a promising prognostic indicator for GBM patients aged $>45$ years (7). In order to develop novel therapeutic strategies to increase overall patient survival, the underlying mechanisms of GBM require to be further elucidated.

Previous studies have explored the signature of deregulated genes for developing effective treatments and better clinical prognostic methods for GBM patients. Bao et al (8) identified a nine-gene signature in glioma patients using the mRNA expression data. An EGFR- and platelet-derived growth factor receptor $\alpha$-centered classification scheme in glioma was established by Sun et al (9). In addition, according to the 2016 World Health Organization (WHO) classification of tumors of the central nervous system, molecular features are incorporated into the classification of GBM (10). In the present study, differentially expressed genes (DEGs) were identified from microarray data downloaded from The Cancer Genome Atlas (TCGA) and Gene Expression 
Omnibus (GEO) databases. Based on the co-expression network of prognosis-associated DEGs, a prognostic prediction system was constructed using Bayes discriminant analysis. Subsequently, the system established in the present study was validated using microarray data from the TCGA dataset, another GEO dataset and a Chinese Glioma Genome Atlas (CGGA) dataset.

\section{Materials and methods}

Microarray data. The mRNA expression data for GBM were downloaded from TCGA database (https://gdc-portal.nci.nih. gov/) on 25th Dec 2016, including 154 tumor samples (survival time information was available for 152 samples) and 13 normal samples. The normal samples were collected from some of the 154 patients with GBM. The Illumina HiSeq 2000 RNA Sequencing platform was used. The genes were identified from the mRNAs in the downloaded dataset using the Human Gene Organization Gene Nomenclature Committee website (http://www.genenames.org/).

Another GBM microarray dataset, GSE22866, was downloaded from the GEO database (http://www.ncbi.nlm. nih.gov/geo/query/acc.cgi?acc=GSE22866), which contained 40 tumor samples and 6 corresponding normal samples. The raw data in the dataset were annotated to obtain the gene expression levels and the average expression values of probes were considered as the expression values of the corresponding genes. Next, the expression values of the genes were subjected to $\log 2$ transformation and normalization using the Limma package in $\mathrm{R}$ language (11). The clinical characteristics of the subjects from which the TCGA and GSE22866 datasets were derived are summarized in Table I.

Screening for DEGs. DEGs between tumor and normal samples were identified in the TCGA and GEO datasets using the Limma and multitest package from Bioconductor (http://bioconductor.org/) in R language $(11,12)$. False discovery rate $<0.5$ and $\mid \log 2$ fold change $(\mathrm{FC}) \mid>1$ were set as the cut-off criteria. The DEGs overlapping between the two datasets were selected for further analysis. The top 50 overlapping DEGs based on the size of their $\mid \log 2 \mathrm{FCl}$ values were subjected to bidirectional clustering.

Identification of prognosis-associated DEGs. Cox regression analysis in the survival package (13) was utilized to select the prognosis-associated genes from the overlapping DEGs, based on the expression values and survival status data. $\mathrm{P}<0.05$ was set as a strict threshold. The top 6 DEGs based on their log-rank P-values were screened as the prognosis-associated DEGs, and were numbered according to their log-rank P-values. Kaplan-Meier survival analysis was performed for the top 6 prognosis-associated DEGs.

Co-expression network of prognosis-associated DEGs. Correlation coefficients (r) between these prognosis-associated DEGs were calculated using the cor function in $\mathrm{R}$ language. The DEG interaction pairs with coefficients of $|r| \geq 0.6$ and $\mathrm{P}<0.05$ were selected to construct a co-expression network, which was visualized using Cytoscape2.8.0 (http://www. cytoscape.org/). Functional modules in the co-expression network were identified using the GraphWeb tool (http://biit. cs.ut.ee/graphweb/) (14).

Construction of a prognostic prediction system. The 152 GBM tumor samples from TCGA were stratified into two groups based on good prognosis and bad prognosis. The good prognosis group was comprised of the samples from patients that were alive and those with a survival time of $\geq 15$ months following sample collection, while the bad prognosis group was comprised of the samples from deceased patients and those with a survival time of $<15$ months. Based on this grouping, Bayes discriminant analysis was performed to analyze the genes in the co-expression network constructed for the prognosis-associated DEGs by using the discriminant Bayes function in $\mathrm{R}$ language $(15,16)$. The discriminant coefficient under the highest discriminant accuracy was considered as the prognostic score. The prognostic DEGs were assembled randomly to genesets to identify the prognostic discriminant. The effectiveness of the prognostic prediction system was evaluated by the receiver operating characteristic (ROC) curve using the pROC package in R3.4.1 (https://cran.r-project.org/web/packages/pROC/index. html). The genes in the highest prognostic discriminant geneset were considered to be signature genes and thereby the constructed system was the prognostic prediction system.

Validation of the prognostic prediction system. To verify the prognostic prediction effect of the constructed system, Kaplan-Meier survival analysis was performed to compare good and bad prognosis groups, which were divided with the scores calculated by the prediction system established in the present study according to the expression level of the signature genes. Next, the microarray dataset GSE13041, containing 191 GBM tumor samples with survival data, was downloaded from the GEO database for further validation of the prediction system. The expression values of the signature genes in this dataset were subjected to analysis with the prediction system for distinguishing different samples based on their prognostic score. Kaplan-Meier survival analysis was also performed to compare the survival status of the two groups to determine the prognostic efficacy of the system. In addition, PartA expression profiles, including 128 GBM tumor samples with survival data, were downloaded from the CGGA database for further validation. Clinical features of patients from which the CGGA dataset was derived are listed in Table I. A similar analysis as for the GSE13041 dataset was performed for validation of the prediction system.

Co-expression network of signature genes. Based on the expression values of these signature genes in TCGA dataset, $r$ values between these signature genes were calculated using the cor function in $\mathrm{R}$ 3.4.1 language (https://stat.ethz. ch/R-manual/R-devel/library/stats/html/cor.html). These gene interaction pairs with coefficients of $|r| \geq 0.6$ and $\mathrm{P}<0.05$ were collected to construct the co-expression network of these signature genes.

Function and pathway enrichment. Function and pathway enrichment was performed for the hub genes in the signature genes co-expression network using the cluster Profiler package in $\mathrm{R}$ language (https://bioconductor.org/packages/release/bioc/html/clusterProfiler.html) (17). 
Table I. Clinical characteristics of patients from the TCGA, CGCA and GEO GSE13041 datasets.

\begin{tabular}{|c|c|c|c|c|}
\hline \multirow[b]{2}{*}{ Clinical characteristic } & \multicolumn{2}{|c|}{ TCGA $(n=172)$} & \multirow{2}{*}{$\begin{array}{c}\text { CGCA } \\
(\mathrm{n}=126)\end{array}$} & \multirow{2}{*}{$\begin{array}{c}\text { GEO } \\
\text { GSE13041 } \\
(\mathrm{n}=191)\end{array}$} \\
\hline & Tumor & Normal & & \\
\hline Age (years) & $59.84 \pm 13.54$ & $54.62 \pm 12.27$ & $46.99 \pm 12.04$ & $53.83 \pm 13.65$ \\
\hline Gender (male/female/undefined) & $99 / 54 / 1$ & $8 / 5 / 5$ & $78 / 48$ & $117 / 74$ \\
\hline Survival status (dead/alive/unknown) & $102 / 40 / 12$ & $12 / 1 / 5$ & $89 / 37$ & $176 / 15$ \\
\hline Overall survival time (months) & $12.06 \pm 10.41$ & $17.09 \pm 11.89$ & $14.49 \pm 8.05$ & $19.37 \pm 19.41$ \\
\hline
\end{tabular}

Values are expressed as the mean \pm standard deviation or as n. GEO, Gene Expression Omnibus; TCGA, The Cancer Genome Atlas; CGCA, Chinese Glioma Genome Atlas.
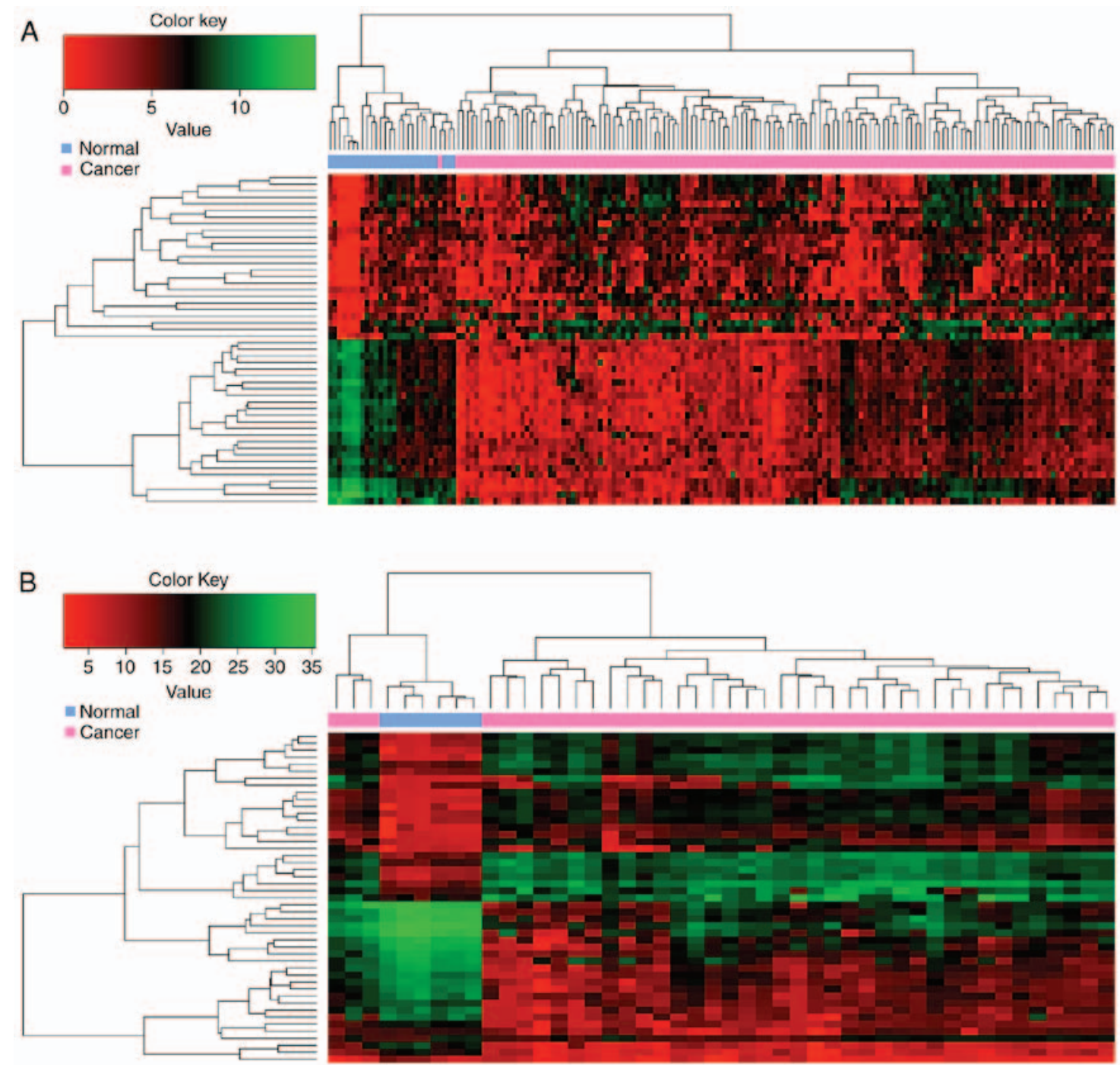

Figure 1. Bidirectional clustering heatmap of the top 50 overlapped differentially expressed genes with high log fold change values. (A) Dataset from The Cancer Genome Atlas; (B) dataset from the Gene Expression Omnibus dataset GSE22866. Tumor samples are marked in pink while normal samples are marked in blue.

\section{Results}

DEG screening. In the present study, 370 and 3564 DEGs were screened from TCGA dataset and the GEO dataset no. GSE22866, respectively. Among them, 288 DEGs over- lapped. The heatmap obtained after bidirectional clustering of the top 50 overlapping DEGs with the highest $\log 2 \mathrm{FCl}$ values is presented in Fig. 1. The expression values of these DEGs were obviously different between normal and GBM samples. 

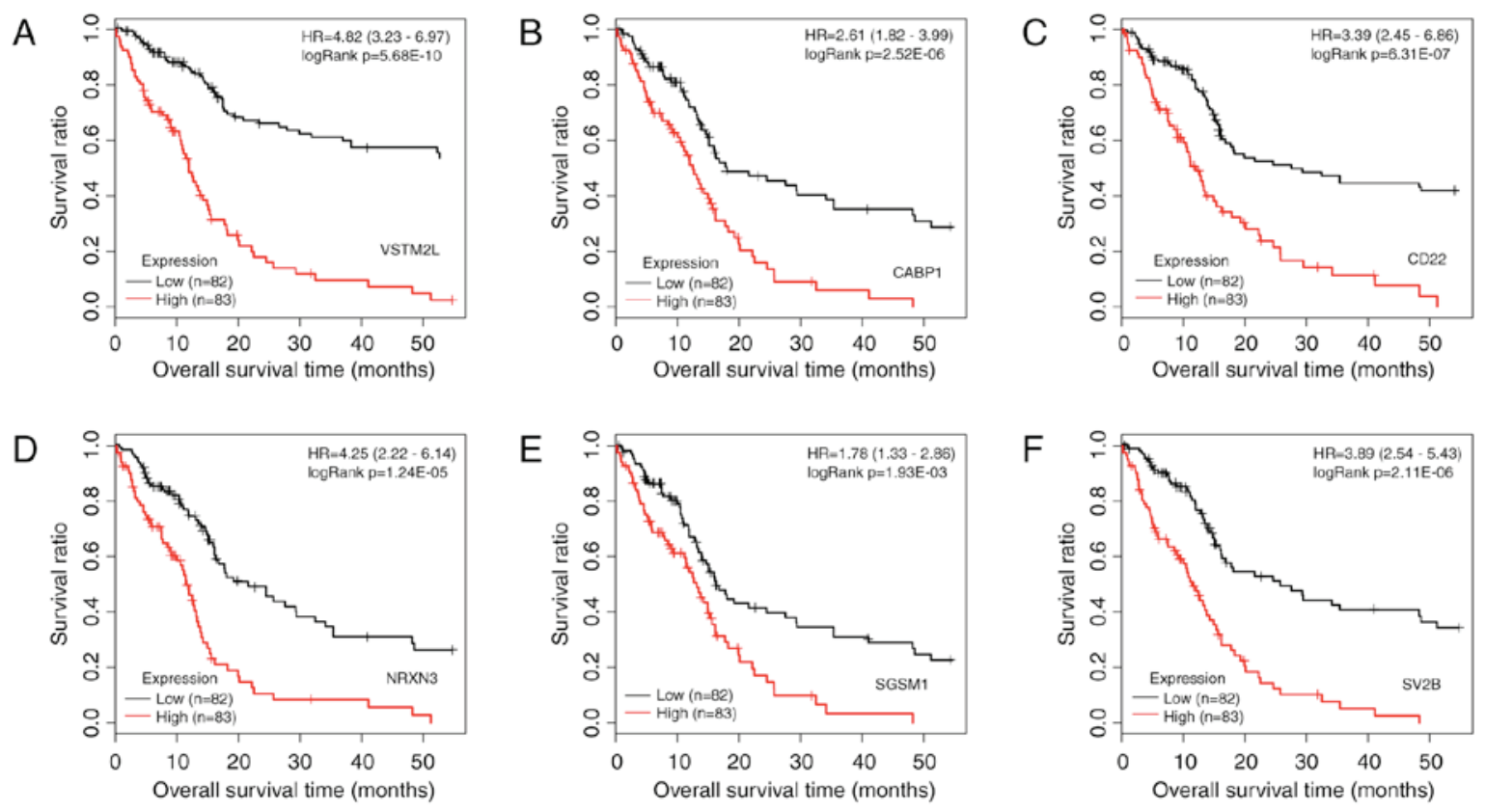

Figure 2. Kaplan-Meier survival curves with patients stratified by high and low expression of the top 6 prognosis-associated differentially expressed genes (A) VSTM2L, (B) CABP1, (C) CD22, (D) NRXN3, (E) SGSM1 and (F) SV2B. The samples with different expression are marked in different colors (red and black). HR, hazard ratio; VSTM2L, V-set and transmembrane domain containing 2 like; CABP1, calcium-binding protein 1; NRXN3, neurexin 3; SGSM1, small $\mathrm{G}$ protein signalling modulator 1 ; SV2B, synaptic vesicle glycoprotein $2 \mathrm{~b}$.

Prognosis-associated DEGs. A total of 123 prognosis-associated DEGs were selected using Cox regression analysis and the log-rank test. These prognosis-associated DEGs were ranked according to their P-values (log-rank test; data not shown). Kaplan-Meier survival curves of the top 6 prognosis-associated DEGs, including V-set and transmembrane domain containing 2-like, calcium-binding protein 1, CD22, neurexin 3, small G protein signaling modulator 1 and synaptic vesicle glycoprotein $2 \mathrm{~b}$, are presented in Fig. 2. The difference in expression of the top 6 genes between tumor tissue and normal tissue are presented in Fig. 3. All of these DEGs were able to distinguish between groups with different survival status $(\mathrm{P}<0.05)$.

Co-expression network of prognosis-associated DEGs. The co-expression network was comprised of 1405 interaction pairs of 112 prognosis-associated DEGs (91 upregulated and 21 downregulated DEGs). A total of 6 significant modules were identified in the network (Fig. 4). Functional analysis revealed that the modules were associated with transmission of nerve impulses (red), nervous system development (blue), nuclear division (green), synaptic transmission (pink) or further unknown functions (yellow and purple).

Construction of prognostic prediction system. The TCGA dataset included 50 patients with good prognosis and 102 patients with bad prognosis. Based on the co-expression network of prognosis-associated genes, a prognostic prediction system comprising 63 signature genes was constructed using Bayes discriminant analysis. The discriminant accuracy of the prognostic prediction system was identified from the ROC curve by determining the area under the curve (AUC) (Fig. 5). The samples from patients with scores $-3<$ score $<0$ were defined as good prognosis; the samples from patients with scores $0 \leq$ score $<3$ were defined as bad prognosis. The

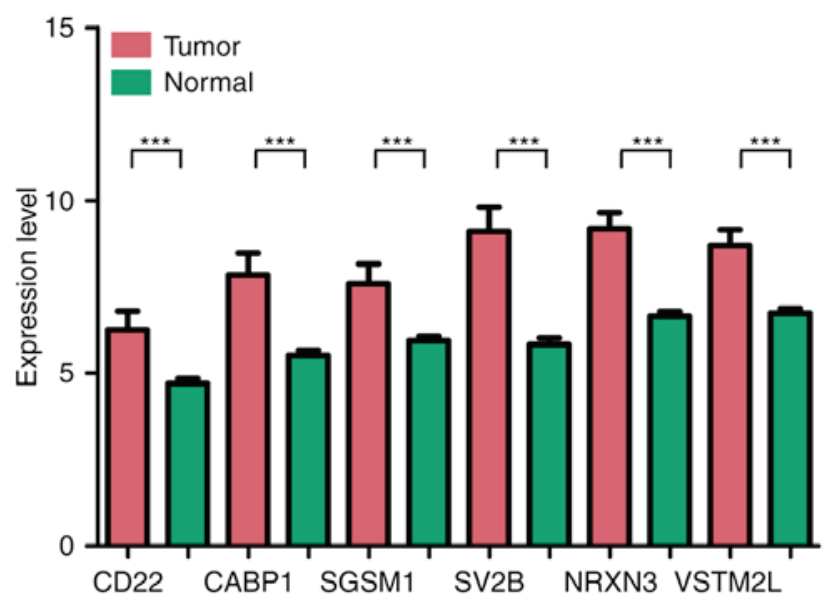

Figure 3. Difference in expression levels of the top 6 genes between tumor tissue and normal tissue. CD22, cluster of differentiation 22; CABP1, calcium binding protein 1; SGSM1, small G protein signaling modulator 1; SV2B, synaptic vesicle glycoprotein 2B; NRXN3, neurexin 3; VSTM2L, V-set and transmembrane domain containing 2 like. ${ }^{* * *} \mathrm{P}<0.05$.

specificity value was 0.929 and the sensitivity value was 0.948 for the ROC curve with the largest AUC of 0.980.

Validation of the prognostic prediction system. In order to validate the performance of the prognostic prediction system, it was tested on the GEO GSE13041 and CGGA datasets. As presented in Fig. 6, based on the expression of the signature genes included in the prognostic prediction system, it was possible to distinguish between samples from patients with good and bad prognosis from the TCGA, GEO GSE13041 and CGGA datasets $\left(\mathrm{P}=1.33 \times 10^{-6}, 0.00534\right.$ and $1.63 \times 10^{-4}$, respectively). The ROC curves of the GSE13041 and CGGA 


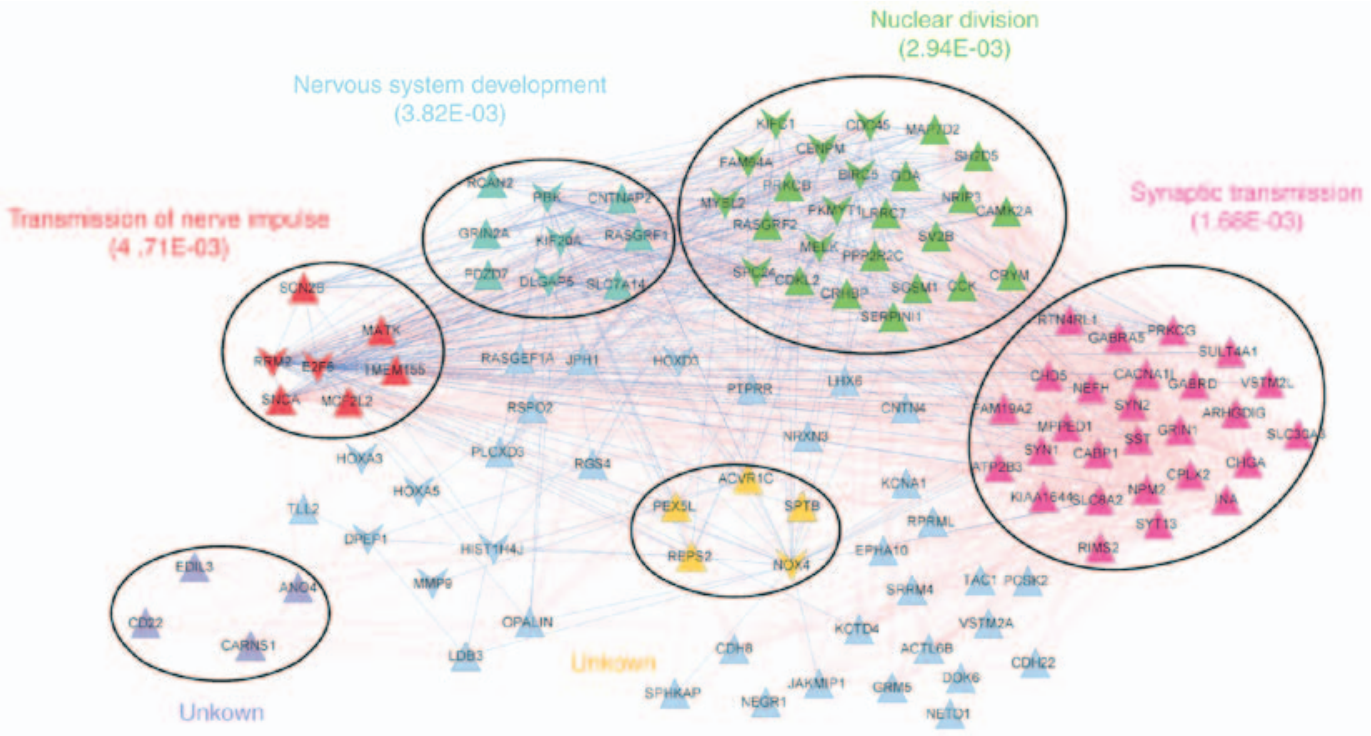

Figure 4. Significant functional modules in the co-expression network of prognosis-associated differentially expressed genes. The numbers are the P-values of genes enriched modules. Blue lines represent a positive connection, while red lines represent a negative connection. Triangles pointing upwards represent upregulated genes, while those pointing downwards indicate downregulated genes.

A

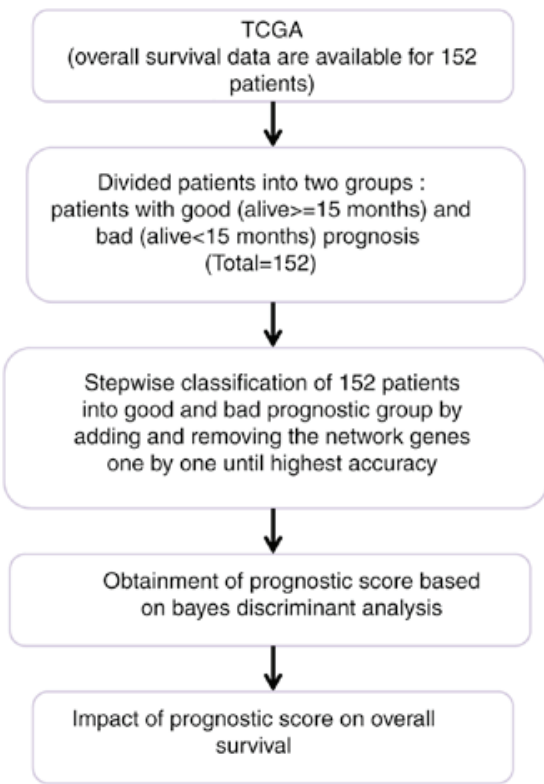

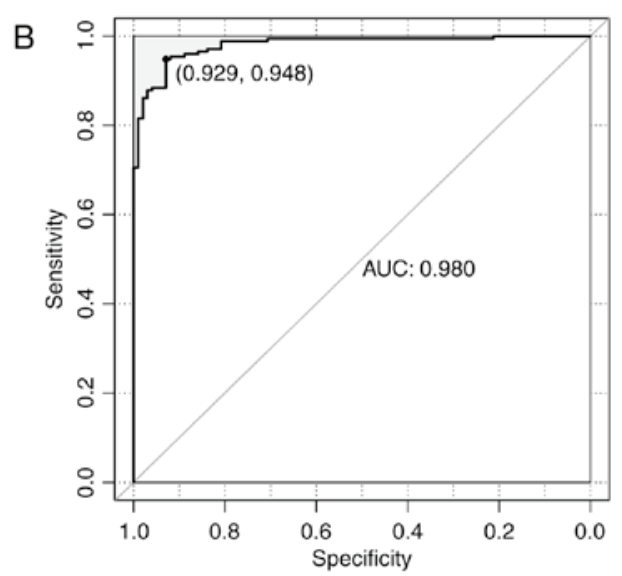

Figure 5. (A) Construction diagram and (B) AUROC curve of the prognostic prediction system. 0.929 was the specificity value and 0.948 was the sensitivity value under the largest AUC of 0.980. AU(RO)C, area under the (receiver operating characteristic) curve; TCGA, The Cancer Genome Atlas.
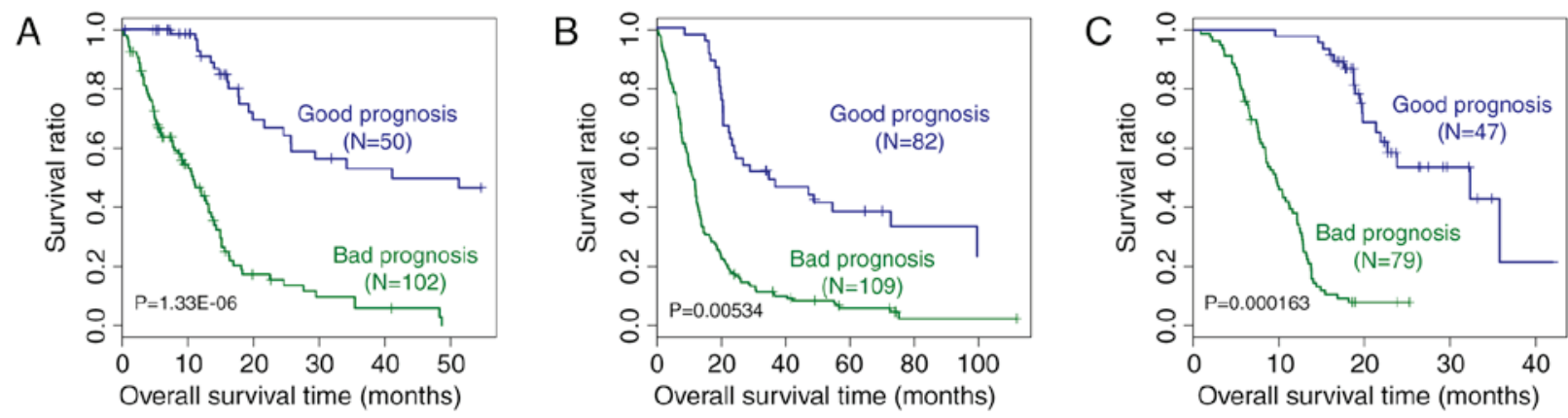

Figure 6. Kaplan-Meier survival curves validating the prediction system established in the present study with the survival compared between the three datasets from (A) The Cancer Genome Atlas, (B) Gene Expression Omnibus (no. GSE13041) and (C) the Chinese Glioma Genome Atlas. Blue lines represent samples from patients with good and green lines represent those from patients with bad prognosis according to the prediction system established in the present study. 

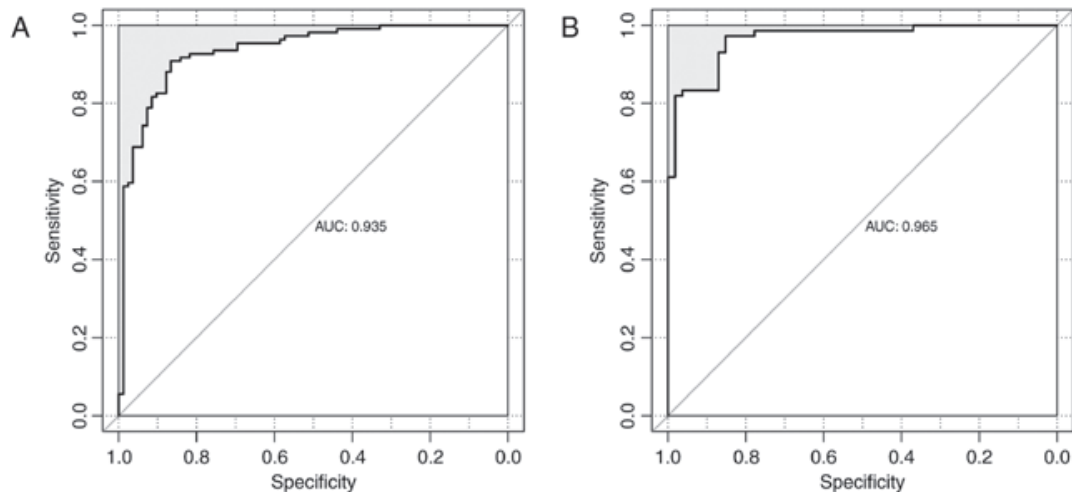

Figure 7. Discriminant receiver operating characteristic curves according to the prognostic prediction system for the microarray data of (A) the Gene Expression Omnibus dataset no. GSE13041 and (B) the Chinese Glioma Genome Atlas dataset. AUC, area under curve.
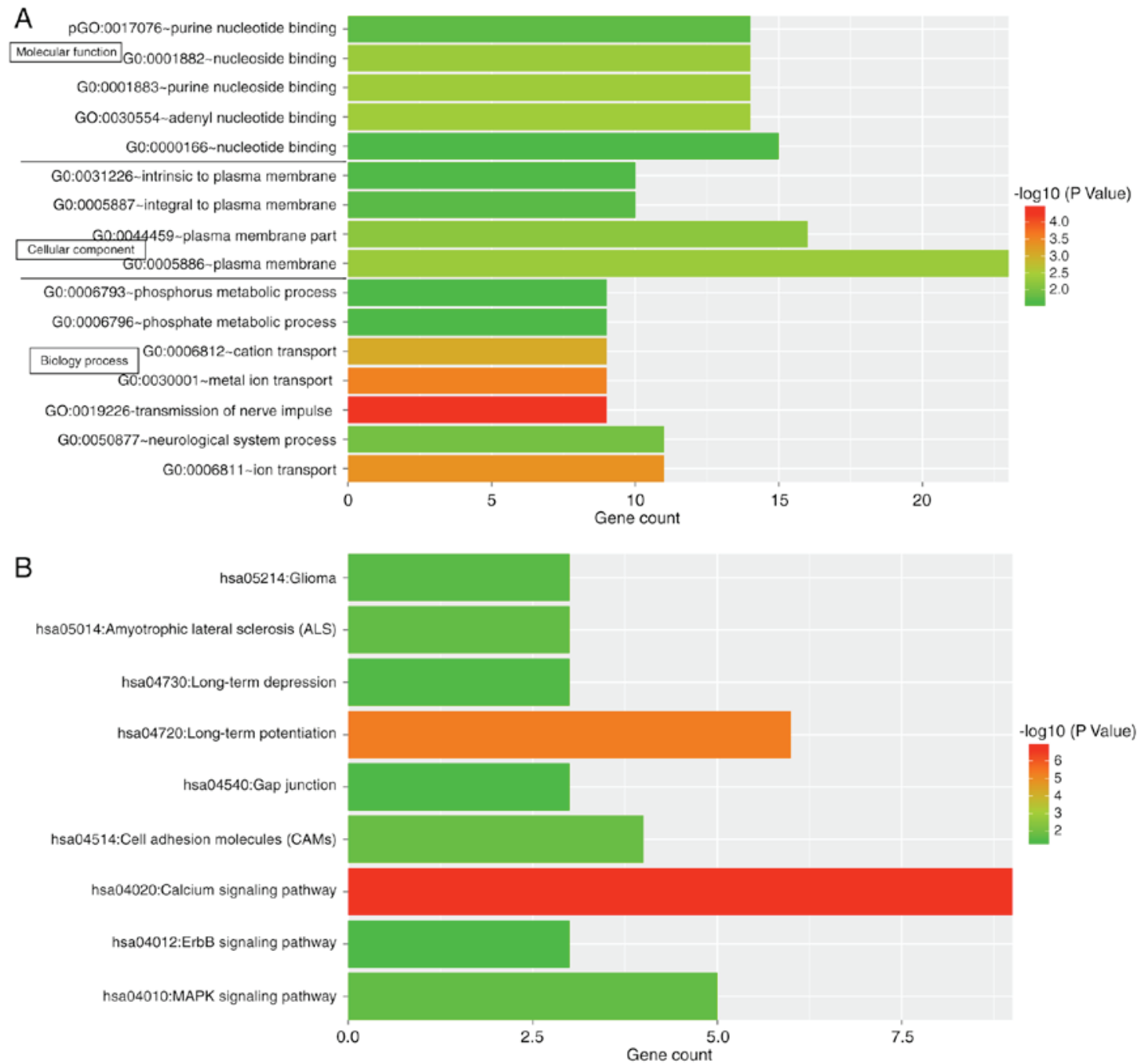

Figure 8. Significant functional enrichment and pathway terms of the signature genes in (A) GO terms and (B) pathways. The number of genes is displayed on the in $\mathrm{x}$-axis, while the associated function/pathway term is displayed on the $\mathrm{y}$-axis. The color is representative of the log2 of the P-value. GO, gene ontology; MAPK, mitogen-activated protein kinase; hsa, Homo sapiens.

datasets are displayed in Fig. 7. The AUC was 0.935 and 0.965, respectively. All of these results proved the accuracy of the prognostic prediction system.

Co-expression network of signature genes. For investigating the possible roles of the 63 prognostic signature genes in GBM, functional and pathway enrichment analysis was performed. The genes were enriched in a total of 16 significant functional terms and 9 significant pathway terms (Fig. 8). The top 3 functional terms included plasma membrane, plasma membrane part and nucleotide binding, while the top 3 pathway terms included calcium signaling pathway, long-term potentiation 


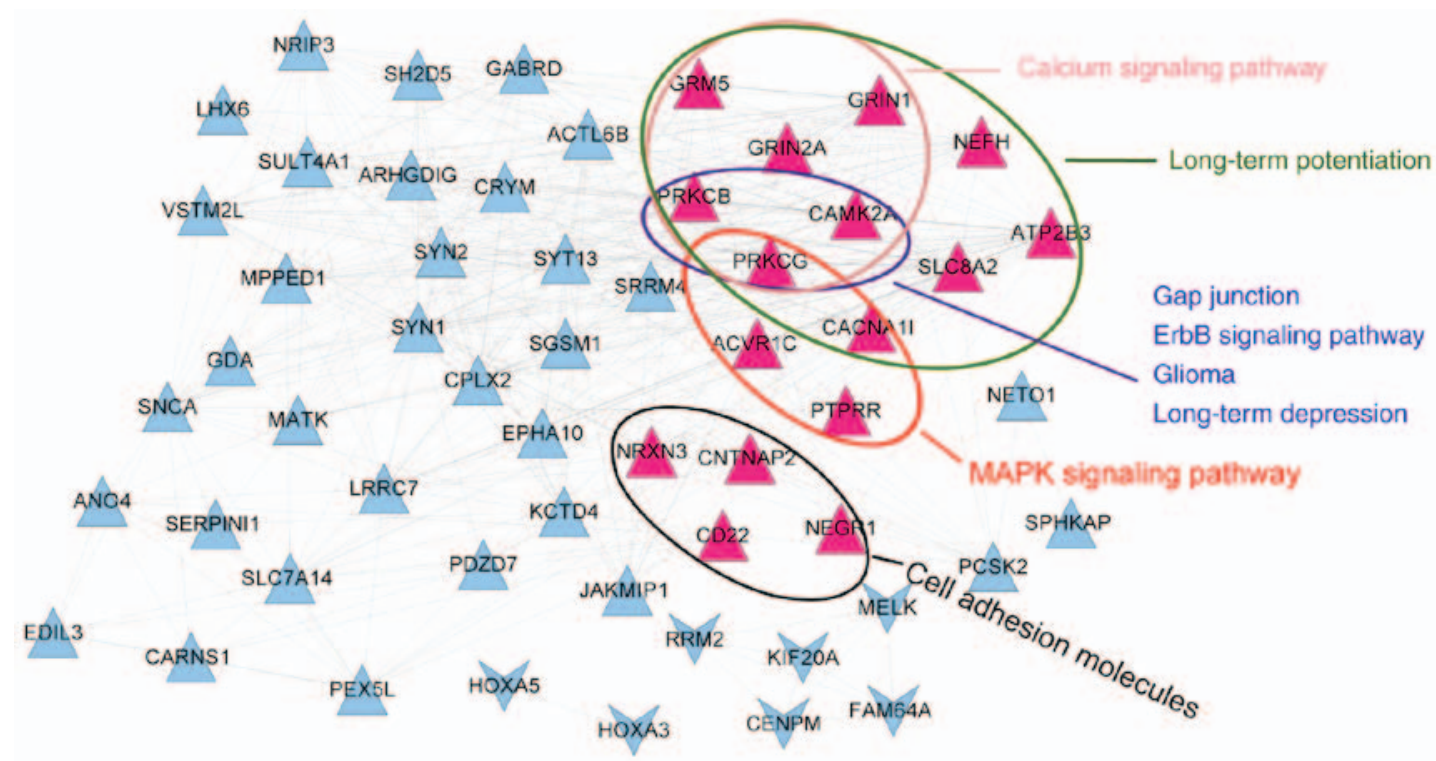

Figure 9. Co-expression network of the signature genes with the significant pathways. Upward-facing triangles represent upregulated genes, while triangles pointing downwards represent downregulated genes. Red triangles represent genes in the significant pathways, and genes in the same circle participate the same pathway. MAPK, mitogen-associated protein kinase.
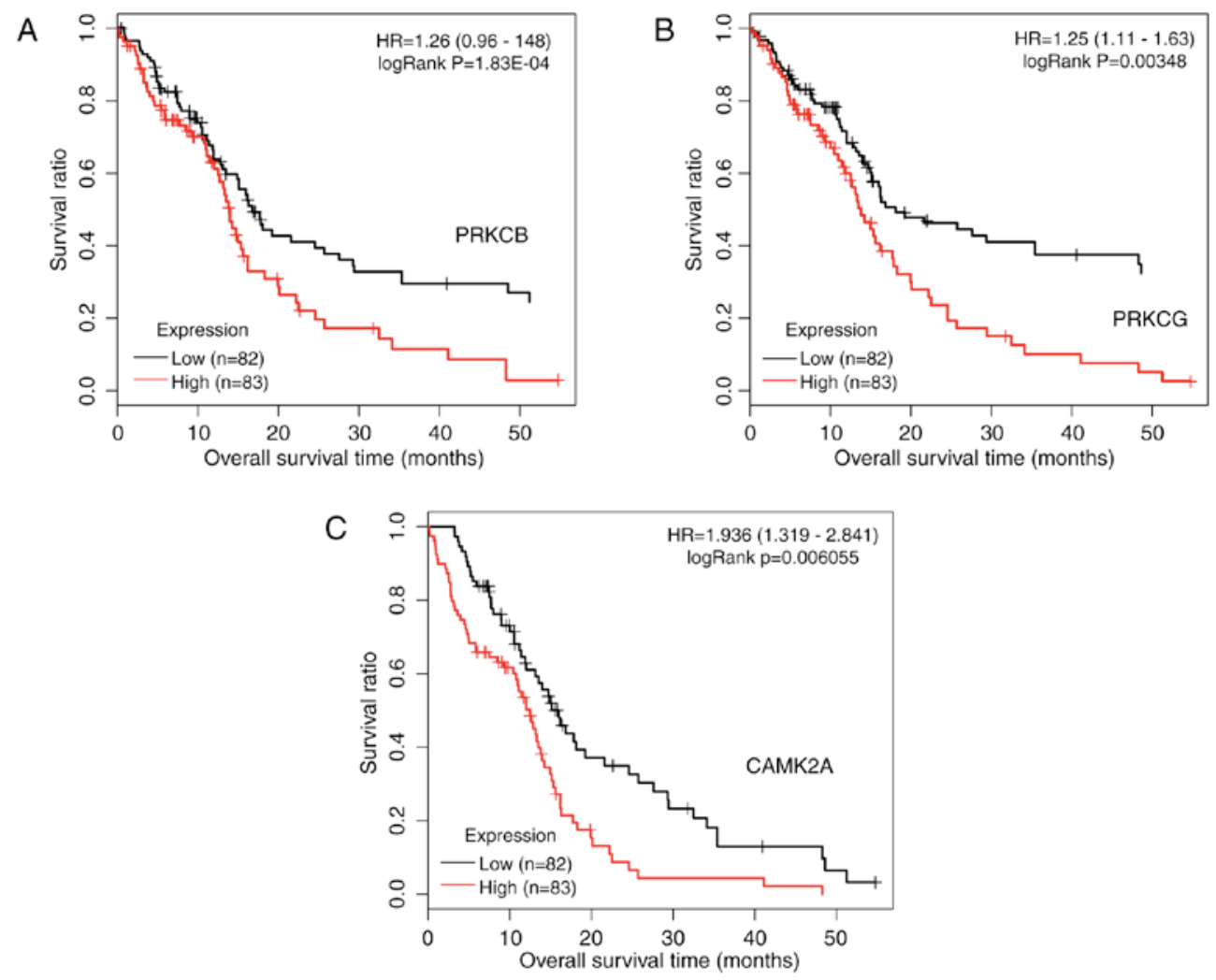

Figure 10. Kaplan-Meier survival curves of three important signature genes: (A) PRKCB, (B) PRKCG and (C) CAMK2A. The samples from patients with different expression of the respective genes are marked in different colors (red and black). HR, hazard ratio; PRKCG, protein kinase C $\gamma$; PRKCB, protein kinase $\mathrm{C} \beta$; CAMK2A, calcium/calmodulin-dependent protein kinase II $\alpha$.

and the mitogen-associated protein kinase (MAPK) signaling pathway.

To gain a better understanding of the associations of these genes with pathways, a co-expression network of signature genes with significant pathways was constructed. The network included 56 genes and 361 interactions. As presented in Fig. 9, protein kinase $\mathrm{C}$ (PKC) $\gamma$ (PRKCG) and PKC $\beta$ (PRKCB) participated in 7 common pathways, and calcium/calmodulin-dependent protein kinase II $\alpha$ (CAMK2A) was involved in 6 pathways. Kaplan-Meier curves with patients stratified according to high or low expression of each of the three genes are presented in Fig. 10. Based on the expression of these three 
genes, it was possible to distinguish between samples with significantly different survival risks.

\section{Discussion}

GBM is a highly aggressive form of brain cancer associated with a poor prognosis. By analyzing the TCGA and GEO GSE22866 datasets, it was identified that they had 288 of the screened DEGs in common. A total of 123 prognosis-associated DEGs were selected from the overlapped DEGs. The co-expression network of the prognosis-associated DEGs was comprised of 1,405 interactions, 91 upregulated DEGs and 21 downregulated DEGs. A total of 6 significant modules were identified in the network, and their significant functions included transmission of nerve impulse, nervous system development, nuclear division and synaptic transmission. The prognostic prediction system was comprised of 63 signature genes with the specificity value of 0.929 and the sensitivity value of 0.948 . The prognostic prediction system was able to distinguish between samples with good and bad prognosis from the TCGA, GEO GSE13041 and CGGA datasets based on the expression of the signature genes $\left(\mathrm{P}=1.33 \times 10^{-6}, 0.00534\right.$ and $1.63 \times 10^{-4}$, respectively). The 63 prognostic signature genes were significantly enriched in 16 significant functional terms and 9 significant pathway terms. The top 3 functional terms included plasma membrane, plasma membrane part and nucleotide binding, while the top 3 pathway terms included calcium signaling pathway, long-term potentiation and the MAPK signaling pathway. In the co-expression network of signature genes with significant pathways, PRKCG and PRKCB were two important genes participating in 7 common significant pathways, while CAMK2A was involved in 6 significant pathways. Based on the expression status of the three genes, it was possible to distinguish between samples with significantly different survival risk.

Calcium mediates long-term potentiation in the hippocampus (18), and is involved in activating the MAPK pathway. In addition, cytosolic calcium regulates ion channels located in the plasma membrane (19). Evidence suggested that calcium signaling has a tumorigenic role in GBM (20). Long-term potentiation is an underlying mechanism for learning and memory. Previous studies have demonstrated that the MAPK pathway is implicated in GBM cell migration and proliferation $(21,22)$. The present study suggested that these signature genes may be associated with the prognosis of GBM patients, partly by modulating the calcium signaling pathway, long-term potentiation and the MAPK signaling pathway.

PRKCG is a susceptibility locus for behavioral disinhibition (23). PRKCG encodes the PKC family $\gamma$ isoform, which normally only occurs in the nervous system. PRKCG is the receptor of phorbol esters, which functions as a class of tumor promoter (24). Louhimo et al (25) reported that Homo sapiens microRNA-23a has a survival effect and its target PRKCG participates in GBM progression-associated processes. It has been reported that PRKCG mutations in spinocerebellar ataxia type 14 affect $\mathrm{C} 1$ domain accessibility and kinase activity, leading to aberrant MAPK signaling (26). PRKCG was reported to be mutated in spinocerebellar ataxia, causing aberrant MAPK signaling (26). The MAPK signaling pathway is involved in the migration and proliferation of GBM cells (21), and MAPK/extracellular signal-regulated kinase signaling activity is comprised of the migration and invasion ability of glioma cells (27). The MAPK signaling pathway also participates in the cellular activity of survival or death (28). Activation of the RAS-MAPK pathway is associated with poor prognosis in neuroblastoma tumors (29). In the present study, it was therefore inferred that PRKCG may affect the prognosis of GBM by the influencing MAPK signaling pathway.

PRKCB also belongs to the PKC family and is considered as a tumor promoter gene, as it enhances certain cellular signaling pathways (30). PRKCB modulates the rate of autophagy, which serves as a pro-death or pro-survival mechanism (31). The PKC family also participates in several cell life and survival-associated processes, including the regulation of cell survival and apoptosis (32). Upregulation of PRKCB is considered beneficial and was identified to be associated with relapse-free survival of breast cancer patients (33). PRKCB is aberrantly expressed in GBM and its expression levels have been reported to be proportional to patient survival time (34). Hence, PRKCB may be a potential prognostic indicator for GBM.

CAMK2A encodes an enzyme involved in calcium-calmodulin-dependent activity. Calcium/calmodulin-dependent protein kinases participate in activating anti-apoptotic signaling pathways and regulating the cell cycle (35). In the present study, CAMK2A was identified to be significantly enriched in long-term potentiation and calcium signaling pathways. This indicates that CAMK2A may affect the prognosis of GBM patients, partly by modulating long-term potentiation and calcium signaling pathways.

The 2016 WHO Classification of Tumors of the Central Nervous system introduced molecular parameters for grouping tumors (10). It is expected that thereby, the accuracy in the diagnosis as well as prognosis of patients may be improved. In the present study, PRKCG, PRKCB and CAMK2A were identified as potential prognostic factors for GBM. The use of PRKCG, PRKCB and CAMK2A as novel molecular markers for GBM may lead to an improvement in prognostic accuracy. Of note, the present study had certain limitations. First, the sample size of patients was limited. Furthermore, the present study focused on bioinformatics analyses only. In vivo and in vitro experiments are required to verify the results of the present study.

In conclusion, the present study established an effective prognostic prediction system and validated its prognostic performance for GBM. PRKCG, PRKCB and CAMK2A may be potential prognostic factors for GBM.

\section{Competing interests}

The authors declare that they have no competing interests.

\section{References}

1. Ricard D, Idbaih A, Ducray F, Lahutte M, Hoang-Xuan K and Delattre JY: Primary brain tumours in adults. Lancet 379: 1984-1996, 2012.

2. Catt S, Chalmers A and Fallowfield L: Psychosocial and supportive-careneeds in high-grade glioma. Lancet Oncol 9: 884-891, 2008

3. Smoll NR, Schaller K and Gautschi OP: Long-term survival of patients with glioblastoma multiforme (GBM) JClin Neurosci 20: 670-675, 2013.

4. Drappatz J, Norden AD and Wen PY: Therapeutic strategies for inhibiting invasion in glioblastoma. Expert Rev Neurother 9: 519-534, 2009. 
5. Thakkar JP, Dolecek TA, Horbinski C, Ostrom QT, Lightner DD, Barnholtz-Sloan JS and Villano JL: Epidemiologic and molecular prognostic review of glioblastoma. Cancer Epidemiol Biomarkers Prev 23: 1985-1996, 2014

6. Gan HK, Kaye AH and Luwor RB: The EGFRvIII variant in glioblastoma multiforme. J Clin Neurosci 16: 748-754, 2009.

7. Srividya MR, Thota B, Shailaja BC, Arivazhagan A, Thennarasu K, Chandramouli BA, Hegde AS and Santosh V: Homozygous 10q23/PTEN deletion and its impact on outcome in glioblastoma: A prospective translational study on a uniformly treated cohort of adult patients. Neuropathology 31: 376-383, 2011

8. Bao ZS, Li MY, Wang JY, Zhang CB, Wang HJ, Yan W, Liu YW, Zhang W, Chen L and Jiang T: Prognostic value of a nine-gene signature in glioma patients based on mRNA expression profiling. CNS Neurosci Ther 20: 112-118, 2014.

9. Sun Y, Zhang W, Chen D, Lv Y, Zheng J, Lilljebjörn H, Ran L, Bao Z, Soneson C, Sjögren HO, et al: A glioma classification scheme based on coexpression modules of EGFR and PDGFRA. Proc Natl Acad Sci USA 111: 3538-3543, 2014.

10. Wen PY and Huse JT: 2016 World Health Organization classification of central nervous system tumors. Continuum (Minneap Minn) 23: 1531-1547, 2017.

11. Li H, Yu B, Li J, Su L, Yan M, Zhang J, Li C, Zhu Z and Liu B: Characterization of differentially expressed genes involved in pathways associated with gastric cancer. PloS One 10: e0125013, 2015.

12. Gu C and Shen T: cDNA microarray and bioinformatic analysis for the identification of key genes in Alzheimer's disease. Int J Mol Med 33: 457-461, 2014.

13. Wang P, Wang Y, Hang B, Zou X and Mao JH: A novel gene expression-based prognostic scoring system to predict survival in gastric cancer. Oncotarget 7: 55343-55351, 2016.

14. Reimand J, Tooming L, Peterson H, Adler P and Vilo J: GraphWeb: Mining heterogeneous biological networks for gene modules with functional significance. Nucleic Acids Res 36 (Web Server issue): W452-W459, 2008.

15. Joutsijoki H, Haponen M, Rasku J, Aalto-Setälä K and Juhola M Error-correcting output codes in classification of human induced pluripotent stem cell colony images. Biomed Res Int 2016: $3025057,2016$.

16. Voigt AP, Eidenschink Brodersen L, Pardo L, Meshinchi S and Loken MR: Consistent quantitative gene product expression: \#1. Automated identification of regenerating bone marrow cell populations using support vector machines. Cytometry A 89: 978-986, 2016

17. Yu G, Wang LG, Han Y and He QY: clusterProfiler: An R package for comparing biological themes among gene clusters. OMICS 16: 284-287, 2012

18. Park P, Sanderson TM, Amici M, Choi SL, Bortolotto ZA, Zhuo M, Kaang BK and Collingridge GL: Calcium-permeable AMPA receptors mediate the induction of the protein kinase a-dependent component of long-term potentiation in the hippocampus. J Neurosci 36: 622-631, 2016.

19. Schroeder JI and Hagiwara S: Cytosolic calcium regulates ion channels in the plasma membrane of Vicia faba guard cells. Nature 338: 427-430, 1989

20. Leclerc C, Haeich J, Aulestia FJ, Kilhoffer MC, Miller AL, Néant I, Webb SE, Schaeffer E, Junier MP, Chneiweiss H and Moreau M: Calcium signaling orchestrates glioblastoma development: Facts and conjunctures. Biochim Biophys Acta 1863: 1447-1459, 2016.

21. Zohrabian VM, Forzani B, Chau Z, Murali R and Jhanwar-Uniyal M: Rho/ROCK and MAPK signaling pathways are involved in glioblastoma cell migration and proliferation. Anticancer Res 29: 119-123, 2009.
22. Sangpairoj K, Vivithanaporn P, Apisawetakan S Chongthammakun S, Sobhon P and Chaithirayanon K: RUNX1 regulates migration, invasion, and angiogenesis via p38 MAPK pathway in human glioblastoma. Cell Mol Neurobiol 37: 1243-1255, 2017.

23. Schlaepfer IR, Clegg HV, Corley RP, Crowley TJ, Hewitt JK, Hopfer CJ, Krauter K, Lessem J, Rhee SH, Stallings MC, et al: The human protein kinase $\mathrm{C}$ gamma gene (PRKCG) as a susceptibility locus for behavioral disinhibition. Addict Biol 12: 200-209, 2007.

24. Geiges D, Meyer T, Marte B, Vanek M, Weissgerber G, Stabel S, Pfeilschifter J, Fabbro D and Huwiler A: Activation of protein kinase C subtypes alpha, gamma, delta, epsilon, zeta and eta by tumor-promoting and nontumor-promoting agents. Biochem Pharmacol 53: 865-875, 1997.

25. Louhimo R, Aittomäki V, Faisal A, Laakso M, Chen P, Ovaska K, Valo E, Lahti L, Rogojin V, Kaski S and Hautaniemi S: Systematic use of computational methods allows stratifying treatment responders in glioblastoma multiforme. Proceedings of the CAMDA Conference. Critical Assessment of Massive Data Analysis, 2011. Systems Biomedicine 1: 130-136, 2013.

26. Verbeek DS, Goedhart J, Bruinsma L, Sinke RJ and Reits EA: PKC gamma mutations in spinocerebellar ataxia type 14 affect $\mathrm{C} 1$ domain accessibility and kinase activity leading to aberrant MAPK signaling. J Cell Sci 121: 2339-2349, 2008.

27. Thomas SL, Alam R, Lemke N, Schultz LR, Gutiérrez JA and Rempel SA: PTEN augments SPARC suppression of proliferation and inhibits SPARC-induced migration by suppressing SHC-RAF-ERK and AKT signaling. Neuro Oncol 12: 941-955, 2010.

28. Dhillon AS, Hagan S, Rath O and Kolch W: MAP kinase signalling pathways in cancer. Oncogene 3279-3290, 2007.

29. Eleveld TF, Schild L, Ebus ME, van Sluis PG, Westerhout EM, Caron HN, Koster JJB, Versteeg R and Molenaar JJ: Abstract A31: Activation of the RAS-MAPK pathway in primary neuroblastoma tumors is associated with poor prognosis. Cancer Res 76 (5 Suppl), 2016.

30. Martiny-Baron G and Fabbro D: Classical PKC isoforms in cancer. Pharmacol Res 55: 477-486, 2007.

31. Wang K and Klionsky DJ: Mitochondria removal by autophagy. Autophagy 7: 297-300, 2011.

32. Bononi A, Agnoletto C, De Marchi E, Marchi S, Patergnani S, Bonora M, Giorgi C, Missiroli S, Poletti F, Rimessi A and Pinton P: Protein kinases and phosphatases in the control of cell fate. Enzyme Res 2011: 329098, 2011

33. Roessler J, Ammerpohl O, Gutwein J, Steinemann D Schlegelberger B, Weyer V, Sariyar M, Geffers R, Arnold N, Schmutzler R, et al: The $\mathrm{CpG}$ island methylator phenotype in breast cancer is associated with the lobular subtype. Epigenomics 7: 187-199, 2015.

34. Hwang E, Yoo KC, Kang SG, Kim RK, Cui YH, Lee HJ, Kim MJ, Lee JS, Kim IG, Suh Y and Lee SJ: PKC $\delta$ activated by c-MET enhances infiltration of human glioblastoma cells through NOTCH2 signaling. Oncotarget 7: 4890-4902, 2016.

35. Rodriguez-Mora O, LaHair MM, Howe CJ, McCubrey JA and Franklin RA: Calcium/calmodulin-dependent protein kinases as potential targets in cancer therapy. Expert Opin Ther Targets 9: 791-808, 2005.

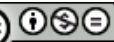

This work is licensed under a Creative Commons Attribution-NonCommercial-NoDerivatives 4.0 International (CC BY-NC-ND 4.0) License. 